\title{
Charged current universality problem and NuTeV anomaly: is SUSY to blame?
}

\author{
Andriy Kurylov*, Michael Ramsey-Musolf ${ }^{* \dagger}$ and Shufang Su${ }^{*}$ \\ * California Institute of Technology, Pasadena, CA 91125, USA \\ ${ }^{\dagger}$ Department of Physics, University of Connecticut, Storrs, CT 06269, USA
}

\begin{abstract}
.
We compute the complete one-loop contributions to low-energy charged current weak interaction observables in the Minimal Supersymmetric Standard Model (MSSM). We obtain the constraints on the MSSM parameter space which arise when precision low-energy charged current (CC) data are analyzed in tandem with measurements of the muon anomaly. The data imply a pattern of mass splittings among first and second generation sleptons and squarks which contradicts predictions of widely used models for supersymmetry breaking mediation. We also discuss the implications of these constraints on the SUSY one-loop contributions to the (anti)neutrino-nucleus deep inelastic scattering. We consider the ratios of neutral current to charged current cross sections, and compare with the deviations of these quantities from the Standard Model predictions implied by the recent $\mathrm{NuTeV}$ measurement. We discuss one scenario in which a right-sign effect arises, and show that it is ruled out by the $\mathrm{CC}$ data. We also study $\mathrm{R}$ parity-violating contributions. Although such effects can account for the violation of the first row CKM unitarity, they can not reproduce the NuTeV anomaly. If $\mathrm{NuTeV}$ anomaly is ultimately explained within the SM, R parity-violating resolution of the CKM unitarity problem can be tested in parity-violating electron scattering experiments at SLAC and TJNAF.
\end{abstract}

\section{INTRODUCTION}

The universality of the charged current weak interaction (CCWI) is an important feature of the Standard Model (SM). The presence of a common coupling strength and $(V-A) \times(V-A)$ current-current interaction structure for all CCWI processes has been tested with high precision in a number of leptonic and semileptonic experiments. The results place significant limits on scenarios for physics beyond the SM which may generate breakdowns of CCWI universality. In the first part of this talk, we report our findings regarding implications of universality tests for minimal supersymmetric extension of the SM (MSSM) with R-parity conservation-one of the leading candidates for "new physics". Supersymmetric theories which break R-parity conservation (equivalently, baryon minus lepton number, $B-L$, conservation) have been considered elsewhere $[1,2,3]$.

Of particular interest for our analysis are the results of superallowed nuclear $\beta$ decays, from which one extracts the Cabibbo-Kobayashi-Maskawa (CKM) quark mixing matrix element $\left|V_{u d}\right|$. When $\left|V_{u d}\right|$ is considered along with the values of $\left|V_{u s}\right|$ and $\left|V_{u b}\right|$ determined from $K_{e 3}$ and $B$-meson decays, respectively, one obtains for the sum of the squares a result falling below the unitarity requirement by $2.2 \sigma[4]$. In what follows, we discuss the implications of this deviation for the MSSM spectrum. 
In the second part of the talk, we discuss the recent result by $\mathrm{NuTeV}$ collaboration, which has performed a precise determination of the ratio $R_{\nu}\left(R_{\bar{\nu}}\right)$ of neutral current (NC) and charged current (CC) deep-inelastic $\nu_{\mu}\left(\bar{\nu}_{\mu}\right)$-nucleus cross sections [5], which can be expressed as $R_{\nu(\bar{\nu})}=\left(g_{L}^{\text {eff }}\right)^{2}+r^{(-1)}\left(g_{R}^{\text {eff }}\right)^{2}$, where $r=\sigma_{\bar{\nu} N}^{C C} / \sigma_{\nu N}^{C C}$ and $\left(g_{L, R}^{\text {eff }}\right)^{2}$ are effective hadronic couplings (defined below). Comparing the SM predictions $[6,7]$ for $\left(g_{L, R}^{\text {eff }}\right)^{2}$ with the values obtained by the $\mathrm{NuTeV}$ Collaboration yields deviations ${ }^{1}$ $\delta R_{\nu(\bar{\nu})}=R_{\nu(\bar{\nu})}^{\exp }-R_{\nu(\bar{\nu})}^{\mathrm{SM}}: \delta R_{\nu}=-0.0033 \pm 0.0007, \delta R_{\bar{\nu}}=-0.0019 \pm 0.0016$

Within the SM, these results may be interpreted as a test of the scale-dependence of the $\sin ^{2} \theta_{W}$ since the $\left(g_{L, R}^{\text {eff }}\right)^{2}$ depend on the weak mixing angle. While the SM prediction for $\sin ^{2} \theta_{w}$ at $\mu=M_{Z}$ has been confirmed with high precision at LEP and SLC, the predicted running of this parameter to lower scales has yet to be studied systematically. The results from the NuTeV measurement imply a $+3 \sigma$ deviation at $\mu \sim 10 \mathrm{GeV}$. This interpretation of the NuTeV results has been the subject of some debate. Unaccounted for QCD effects, such as charge symmetry-breaking in parton distributions or nuclear shadowing[8], have been proposed as possible remedies for the anomaly. Alternatively, one may consider physics beyond the SM, as reviewed in Ref. [9]. In what follows, we focus on one new physics scenario, namely, supersymmetry (SUSY). Here, we carry out a model-independent treatment, avoiding the choice of a specific mechanism for SUSYbreaking mediation. We find that it is difficult - if not impossible - to choose MSSM parameters so as to improve agreement with the $\mathrm{NuTeV}$ result.

\section{MSSM IN A NUTSHELL}

Low energy SUSY is an attractive scenario from a number of standpoints. By introducing a superpartner for every SM particle, it provides a solution to the hierarchy problem associated with Higgs mass renormalization; it produces coupling unification at the GUT scale; and it is a prediction of superstring theory. It remains to be seen, however, which version of SUSY correctly describes electroweak phenomena. In particular, details of the superpartner spectrum (e.g., masses and mixing angles) are largely unknown. Limits on branching ratios obtained from collider data provide, in general, only weak lower bounds.

If R-parity (and, thus, $B-L$ ) is conserved, the SUSY corrections to low energy observables arise only via tiny loop effects. In order to become sensitive to such contributions, one generally requires a precision of $\sim(\alpha / \pi) \times(M / \tilde{M})^{2}$, where $M$ is the relevant mass of a SM particle and $\tilde{M}$ is a superpartner mass. For CCWI observables and NuTeV, one has $M \sim M_{W}$, so that only a few $\times 10^{-3}$ precision is needed to achieve sensitivity. If R-parity is not conserved, new tree-level SUSY effects appear for virtually all observables. A comprehensive review of supersymmetry can be found in Ref. [10].

\footnotetext{
${ }^{1}$ We use the quoted experimental errors on $R_{\nu}$ and $R_{\bar{\nu}}$, rather than adding the errors on $\left(g_{L, R}^{\text {eff }}\right)^{2}$ in quadrature, since the latter are correlated and derived from the experimental cross section ratios.
} 


\section{CHARGED CURRENT UNIVBERSALITY}

Any CCWI amplitude is properly normalized to $G_{\mu}=\sqrt{2} g^{2} /\left(8 M_{W}^{2}\right)\left[1+\Delta r_{\mu}\right]$, the Fermi constant measured in $\mu$-decay, which is one of the three most precise inputs for the gauge sector of the MSSM. Here, $g$ is the universal weak coupling, $M_{W}$ is the $W$-boson mass, and $\Delta r_{\mu}$ includes the effects of weak, radiative corrections in the MSSM as well as other possible new physics contributions to $\mu$-decay. The Fermi constant relevant for light quark $\beta$-decay is $G_{F}^{\beta}=G_{\mu} V_{u d}\left(1-\Delta r_{\mu}+\Delta r_{\beta}\right)$, where $\Delta r_{\beta}$ is the analogue of $\Delta r_{\mu}$. The difference $\Delta r_{\beta}-\Delta r_{\mu}$ is sensitive only to non-universal effects, such as vertex corrections, box diagrams, and external leg corrections. Because it compares these corrections as they appear in leptonic and semileptonic decay amplitudes, $G_{F}^{\beta}$ is essentially a measure of slepton-squark universality in the MSSM. Moreover, since both $\Delta r_{\beta}$ and $\Delta r_{\mu}$ pertain to processes with $e^{+} \nu_{e}\left(e^{-} \bar{\nu}_{e}\right)$ in the final state, the difference $\Delta r_{\beta}-\Delta r_{\mu}$ is considerably more sensitive to effects produced by second generation sleptons than to those produced by the first generation.

Requiring that the non-universal SUSY corrections produce no additional deviation from CKM unitarity (on top of existing 2.2 $\sigma$ ) together with constraints from muon anomalous magnetic moment [11] implies that $\Delta r_{\beta}^{\text {susy }}-\Delta r_{\mu}^{\text {susy }}<0$ at $95 \%$ confidence level. We found that this constraint leads to a non-trival relationship between the masses of the superpartners of the muon $\tilde{\mu}_{L}$ and the first generation squarks $\tilde{q}_{L}$. In particular, one always has $M_{\tilde{\mu}_{L}}>M_{\tilde{q}_{L}}$. This phenomenological solution is particularly interesting from the standpoint of both gauge-mediated and mSUGRA models of SUSY-breaking mediation, which generally predict $M_{\tilde{q}}>M_{\tilde{\ell}}$. In mSUGRA, this hierarchy results from gluino contributions to the renormalization group running of the masses down from the GUT scale. Inverting this hierarchy would presumably require modifying the universality assumptions made for the parameters of $\mathcal{L}_{\text {soft }}$ at the GUT scale.

\section{NUTEV ANOMALY}

We represent the charged and neutral current neutrino-quark interactions by an effective four fermion Lagrangian:

$$
\begin{aligned}
\mathcal{L}_{\nu q}^{N C} & =-\frac{G_{\mu} \rho_{\nu N}^{N C}}{\sqrt{2}} \bar{\nu}_{\mu} \gamma^{\lambda}\left(1-\gamma_{5}\right) \nu_{\mu} \sum_{q} \bar{q} \gamma_{\lambda}\left[2 \epsilon_{L}^{q}\left(1-\gamma_{5}\right) / 2+2 \epsilon_{R}^{q}\left(1+\gamma_{5}\right) / 2\right] q \\
\mathcal{L}_{\nu q}^{C C} & =-\frac{G_{\mu} \rho_{\nu N}^{C C}}{\sqrt{2}} \bar{\mu} \gamma^{\lambda}\left(1-\gamma_{5}\right) \nu_{\mu} \bar{u} \gamma_{\lambda}\left(1-\gamma_{5}\right) d+\text { h.c. }
\end{aligned}
$$

where

$$
\epsilon_{L}^{q}=I_{L}^{3}-Q_{q} \kappa_{\nu} \sin ^{2} \theta_{W}+\lambda_{L}^{q}, \epsilon_{R}^{q}=-Q_{q} \kappa_{\nu} \sin ^{2} \theta_{W}+\lambda_{R}^{q}
$$

The parameters $\rho_{\nu N}^{N C}=\rho_{\nu N}^{C C}=\kappa_{\nu}=1$ and $\lambda_{L, R}^{q}=0$ at tree-level in the SM. These quantities differ from their tree-level values when $\mathcal{O}(\alpha)$ corrections in the SM or MSSM are included or when other new physics contributions arise. The NC to CC cross section 
ratios $R_{\nu}$ and $R_{\bar{\nu}}$ can be expressed in terms of the above parameters via the effective couplings $\left(g_{L, R}^{\text {eff }}\right)^{2}$ in a straightforward way:

$$
\left(g_{L, R}^{\text {eff }}\right)^{2}=\left(\frac{\hat{M}_{Z}^{2}}{\hat{M}_{W}^{2}}\right)^{2}\left(\frac{\hat{M}_{W}^{2}-q^{2}}{\hat{M}_{Z}^{2}-q^{2}}\right)^{2}\left(\frac{\rho_{\nu N}^{N C}}{\rho_{\nu N}^{C C}}\right)^{2} \sum_{q}\left(\epsilon_{L, R}^{q}\right)^{2}
$$

We calculate the MSSM contributions to $\rho_{\nu N}^{N C, C C}, \kappa_{\nu}, \lambda_{L, R}^{q}, R_{\nu}$, and $R_{\bar{\nu}}$ both with and without R-parity conservation. In the former case we randomly choose values for these parameters discarding any points that yield SUSY particle masses below present collider lower bounds or violate constraints from the $Z$-pole electroweak precision measurements. In the latter case we utilize constraints on R-parity violating parameters from superallowed nuclear $\beta$-decay, atomic PV measurements of the cesium weak charge, the $e / \mu$ ratio $R_{e / \mu}$ in $\pi_{l 2}$ decays, and a comparison of the Fermi constant $G_{\mu}$ with the appropriate combination of $\alpha, M_{Z}$, and $\sin ^{2} \theta_{W}$. In both cases, SUSY corrections to $R_{\nu}$ and $R_{\bar{\nu}}$ generally have too small a magnitude and the wrong sign to account for the effect.

\section{CONCLUSIONS}

SUSY loop corrections to CC universality are generically small. They can alleviate the unitarity problem by no more that $0.5 \sigma$ but required parameters are difficult to accommodate in all known models of SUSY breaking. SUSY corrections also tend to worsen the $\mathrm{NuTeV}$ anomaly. If the CKM unitarity problem and NuTeV anomaly are not resolved within the SM (by, e.g. new value of $V_{u s}$ for the former and nuclear effects for the latter), they will pose serious difficulties for considering the MSSM as the true extension of the SM.

\section{REFERENCES}

1. V. Barger and K. Cheung, Phys. Lett. B480, 149 (2000).

2. M.J. Ramsey-Musolf, Phys. Rev. D62:056009 (2000).

3. B.C. Allanach, A. Dedes, and H. Dreiner, Phys. Rev. D60:075014 (1999).

4. I.S. Towner and J.C. Hardy, nucl-th/9809087.

5. G.P. Zeller, et al., the NuTeV Collaboration, Phys. Rev. Lett. 88, 091802 (2002).

6. Review of Particle Properties, Phys. Rev. D 66, 010001 (2002).

7. J. Erler, private communication.

8. G.A. Miller and A.W. Thomas, hep-ex/0204007; see also K.S. McFarland, hep-ex/021001.

9. S. Davidson, et al., JHEP 02, 037 (2002).

10. For a review, see Perspectives on Supersymmetry, G.L. Kane, Ed., World Scientific, Singapore, 1998.

11. Muon g-2 Collaboration, H.N. Brown et al., Phys. Rev. Lett. 86, 2227 (2001). 